\title{
Hand Rejuvenation Using Autologous Fat Transfer
}

\author{
Review Article
}

\section{Mohammad Ali Shakeri Hosseinabad ${ }^{*}$}

1. Resident of Dermatology, Ahvaz Jundishapur University of Medical Sciences, Ahvaz, Iran.

\begin{abstract}
Atrophy and wrinkling of the skin, elevation of bones, and bulging of the veins and tendons are among outstanding characteristics of hand aging. In recent years, there has been an increase in demand for apparent rejuvenation of the hand. A review of the research literature shows that there are many options for rejuvenation of the hand including peeling agents, removal of dead micro-dermal layers, and dermal fillers. The present study was an attempt to consider and describe autologous fat transfer to the hands. In so doing, the databases of PubMed, Google Scholar, and Science Direct were searched, and out of the retrieved 1988 articles, 30 were used in the present review. The analysis carried out by the researchers showed that autologous fat transfer is the most promising method that has recently drawn lots of attention.
\end{abstract}

Keywords: Hand rejuvenation, autologous fat transfer, beauty.

\section{Introduction}

The appearance of human hand shows the real age of human. Research showed that people can estimate someone's real age based on the appearance of their hand (1). Except for the face and the neck, hands are the most observable parts of the body, which are exposed to environmental factors such as ultraviolet, detergent chemicals, and other substances, which accelerates the aging process (2-4). Therefore, it is not surprising if special methods for rejuvenating the appearance of the hands have been proposed over the last decade (5-7). The challenge that the surgeons are faced with, in rejuvenation of hands, is to make the appearance of the hand skin without wrinkles and bumps and increase its clarity. The effect of external factors such as ultraviolet and photoaging factors leads to formation of irregular wrinkles and spots like lentigines, solar purpura, actinic keratosis, seborrheic keratosis, and telangiectasia $(8,9)$. Aging leads to intrinsic effects such as gradual disappearance of subcutaneous volume and tissue atrophy in the form of a decrease in collagen and dehydration in the tissues of the hands $(10,11)$. In this case, it leads to dorsal skin wrinkles, and extensor tendons can be seen more clearly, which causes blue tortuous subcutaneous veins to be seen $(2,12)$. It seems that various options are available for doctors and patients to deal with these factors all of which have unique effects on the appearance of the dorsal part of the hand. Decreasing methods use trichloroacetic acid and phenolic peeling peptides in order to create skin quality by creating thickness of the skin and making changes in bright pigment (13-15). Hand lift surgery removes wrinkles from the back of the hand, wrist, and forearm $(14,16)$.

*Corresponding Author:

Mohammad Ali Shakeri Hosseinabad

Resident of Dermatology,

Ahvaz Jundishapur

University of Medical Sciences, Ahvaz, Iran

Email id: m.shakeri62@yahoo.com
Dermal fillers and volume raisers such as polymethylmethacrylate, calcium hydroxyapatite, hyaluronic acid, poly lactic acid, collagen, and silicone give the hand a bigger and bulkier appearance by reducing looseness and wrinkles of the skin and hiding bulging structures such as bones, tendons, and veins; therefore, new collagens form $(4,6,8,9,27)$. Laser therapies such as Q-switched lasers, intense pulsed light, photodynamic therapy, ablative fractionated lasers, nonablative fractionated lasers, radio frequency, and plasma skin regeneration lead to remarkable effects on the skin including epidermal whiteness, removing visible lesions, improving skin texture, reducing wrinkles, and skin regeneration $(9,18)$. Bulging veins can be treated using sclerotherapy, intravascular laser erosion, and phlebectomy $(9,15)$. Inducing and creating subcutaneous collagen and skin removal are mechanical methods which cause epidermal thickness and elastin sedimentation $(15,19)$.

The present study was aimed at reviewing hand rejuvenation using autologous fat transfer. In so doing, the databases of PubMed, Google Scholar, and Science Direct were searched using different keywords like autologous fat transfer, beauty, plastic surgery, and hand skin, and out of the retrieved 1988 articles published during 2000-2017, 31 articles were used in the present study. After the literature over the last 17 years was reviewed, it was concluded that autologous fat transfer is considered as the best method with minimum complications in rejuvenating the appearance of hands. Fat grafting to the back of hands dates back to 1980, and since then, it has been developing as a safe and reliable method $(2,20)$.

\section{Anatomy of the hands}

Bidic et al (2010) studied dorsal hand anatomy relevant to injection of volumetric and dermal fillers of cosmetics (21). They found that in the dorsal subcutaneous tissue, there are three distinct fatty laminae separated by thin fascia. Dorsal veins and sensory nerves reside within the intermediate lamina 
where extensor tendons are found within the deep part. The most superficial layer of lamina contains no anatomic structure. They concluded that the grafted fat tissue can theoretically be injected into this layer without endangering the veins, sensory nerves, and dorsal extensor tendons.

\section{Fat grafting methods}

Hand rejuvenation methods have been successful; however, none of them have been as successful as autologous fat transfer. Primary reports by Zuk et al (2001) (22) show that stem multicellular cells found in adipose tissue of adults can be used as a primary and important treatment in many beautification methods (23, 24). Removal of adipose tissue (removal of cell or tissue from an individual to whom it is grafted) is carried out in an autologous way, which is quite compatible, and it can be removed whenever it is needed (25). The fat makes the hand volumetric, and causes rejuvenation state to return to the skeletal structure of the hand.

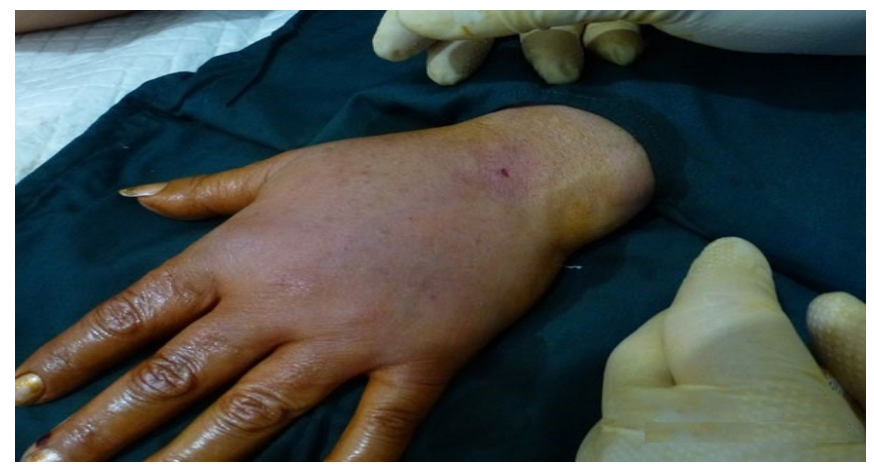

Fig. 1. Autologous fat transfer by dorsal hand injection

Late $1980 \mathrm{~s}$ is considered as the onset of autologous fat transfer as an effective method to preserve the beautiful appearance of hands (9). Fournier was the pioneer of this beautification method and an advocate of injecting a mass of fat into the back of the hand and then massaging it over the entire hand (15). The results of this method; however, were unpredictable, and it was generally considered as an unreliable method $(2,9)$. The method was the same until 1992, but afterwards, Coleman changed it into its today's form which is less complex and causes more satisfaction among patients (9). Following the patient's consent, fat can be taken from different parts of the body including abdomen, flanks, thighs, and inner knees $(9,26)$.

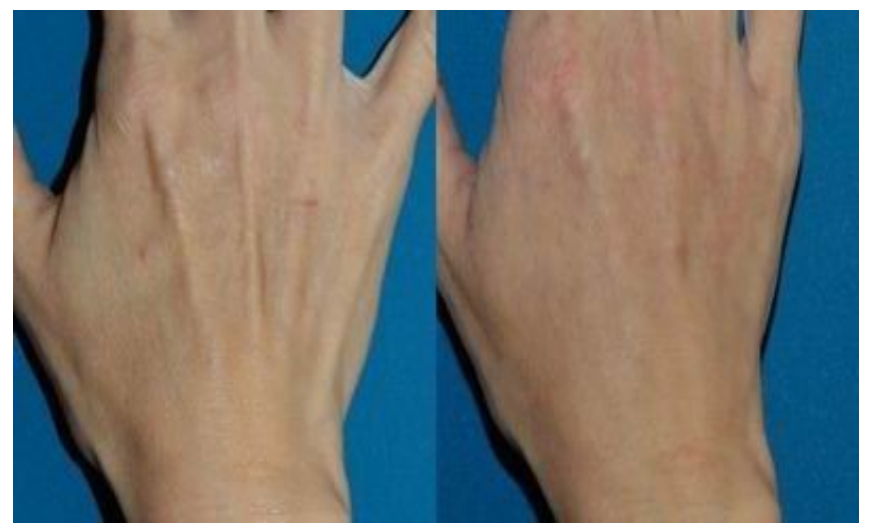

Fig. 2. Autologous fat transfer to the back of the hand
If the part from which the fat is taken is between the inside and outside part of the hip, the patient needs to be marked while he/she is standing. If it is from the external part of the hip or abdominal wall, the individual should be in a supine position (5). To take fat from these parts, lidocaine with epinephrine is usually injected 15 minutes before the fat is taken $(9,13,20)$. To take $15-40 \mathrm{ml}$ fat for each hand, $10 \mathrm{ml}$ tulip syringe with 2 to $3 \mathrm{~mm}$ canola is utilized. This causes the surface and underlying fatty layers are separated in the suction syringe $(2,9,13,14)$. After the fluid is poured into the tissue, the surface fat is emptied with a syringe $(2,9,15)$. The $10 \mathrm{ml}$ syringe that is centrifuged at a speed of 3,000-3,600 rpm for 3 minutes causes accumulation of more fat $(2,9,14)$. The centrifuged fat is more preferred, because an increase in concentration of fat cells leads to longer lifespan and slower absorption of the injected volume $(9,27)$.

The hand is prepared by placing it in a sterilized state. Afterwards, lidocaine is injected to wrinkled parts of the back of the hand and wrists, and at the same time, the hand is massaged a little so it can spread all over the hand $(9,15,19)$. Grafting is carried out using a syringe of 1 to $3 \mathrm{~mm}$ and $1 \mathrm{ml}$ canola with a needle number 11 to 18 the end of which has a shear and modular section so that canola can be released and the risk of blood eruption and bleeding can drop $(2,5,9,20)$.

Sommer and Sattler (2000) reviewed different methods and the rate of their success and concluded that regardless of the method, the best result will be achieved when the lowest volume is used (28). Carpaneda examined this issue with regard to histology and concluded that the diameter of the utilized and injected fat section should be $3 \mathrm{~mm}$ or less so that angiogenesis can occur in it again (29). Coleman (2002) (2) and Fabi and Goldman (2012) (9) supported this method which includes a lot of small tunnels to maximize the level of contact between the adipose tissue and the receptor. This method improves nutrition, respiration, stability, integrity, and formation of the grafted adipose tissue $(2,9)$.

Studies show that about $60 \%$ of grafted cells that were over $1 \mathrm{~mm}$ died due to a decrease in respiratory and nutritional source $(9,29)$. An amount of 10-30 mm fat needs to be injected to the hand so that its puffy appearance can be observable $(9,14,15,20)$. Some adipose should be injected to the basis and beginning of each finger, without which the rate of grafting success will decrease (15). The gap created in the hand can be restored with absorbable sutures. The hand needs to be held up for 24 hours after the surgery, and soft bandage should be used for 5 days $(13,20)$. Most researchers disagree whether the hand should be massaged during the first week after the surgery or not $(2,13)$. The patients usually need to know that swelling and skin color change will continue for 1 to 2 weeks after the surgery. Researchers have suggested consuming antibiotics for 10 days after the surgery (9).

\section{Patients' concerns after adipose tissue grafting}

Although this method is one of the newest methods in aesthetics, as it is reported, it is associated with some problems after the surgery. The reported 
problems include inflammation in subcutaneous connective tissue, infection in the hands and the place of fat removal, creation cysts in patients, and temporary numbness $(15,19,30)$. In one of the studies, $24 \%$ of the patients injected adipose to their hand again (19). In another study, $10 \%$ of the patients re-injected adipose for higher stability (15).

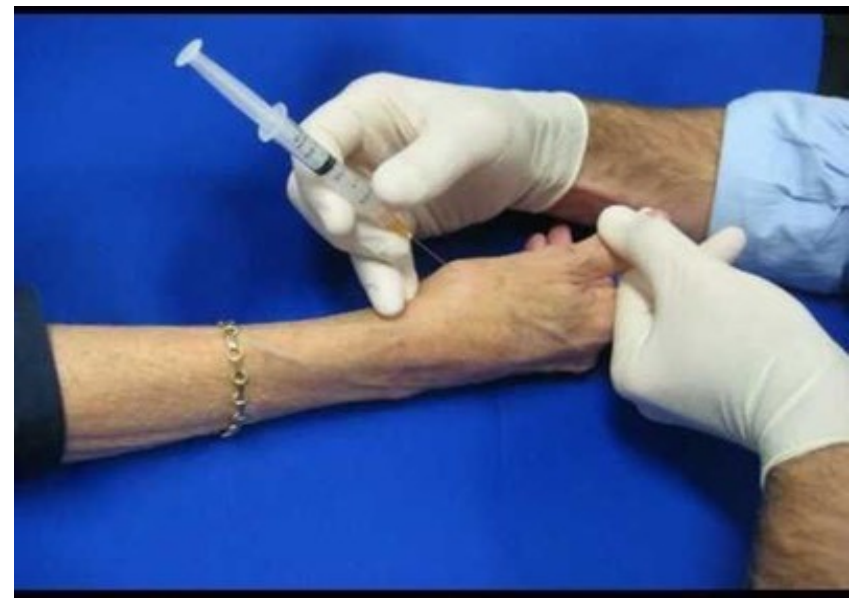

Fig. 3. Extracting secretions to prevent inflation

\section{Conclusion}

It seems that future studies of adipose tissue grafting to hands should use more removed fat stem cells. The place of these cells seems different with regard to their function. Different studies show that in white adipose tissue, the stem cells retrieved from adipose tissue in subcutaneous fat tissue had a higher efficiency than visceral fat tissue which mostly accumulates in brachial adipose tissue $(23,24,31)$. This information can provide researchers with an appropriate insight in their future studies for proper retrieval of fat and then grafting it to the hands. Recent studies showed that when individuals get older, they worry not only about the function of their hands but also about the beauty of them; therefore, it is noteworthy finding methods that give back beauty to hands and do not reduce their performance.

\section{References}

1. Bains RD, Thorpe H, Southern S. Hand aging: patients' opinions. Plastic and reconstructive surgery. 2006;117(7):2212-8.

2. Coleman SR. Hand rejuvenation with structural fat grafting. Plastic and reconstructive surgery. 2002;110(7):1731-44.

3. Jakubietz RG, Kloss DF, Gruenert JG, Jakubietz MG. The ageing hand. A study to evaluate the chronological ageing process of the hand. Journal of Plastic, Reconstructive \& Aesthetic Surgery. 2008;61(6):681-6.

4. Inglefield C. Nonsurgical hand rejuvenation with Dermicol-P35 30G. Aesthetic surgery journal. 2009;29(3 Supplement):S19-S21.

5. Fulton J, Caperton C, Weinkle S, Dewandre L. Filler injections with the blunt-tip microcannula. Journal of drugs in dermatology: JDD. 2012;11 (9):1098-103.
6. Park TH, Yeo KK, Seo SW, Kim J-k, Lee JH, Park $\mathrm{JH}$, et al. Clinical experience with complications of hand rejuvenation. Journal of Plastic, Reconstructive \& Aesthetic Surgery. 2012;65(12):1627-31.

7. Ozden BC, Arinci A, Aydin A, Buyukbabani N. Self -rejuvenation of the hand. Journal of Hand Surgery (European Volume). 2010;35(6):511-2.

8. Kühne U, Imhof M. Treatment of the ageing hand with dermal fillers. Journal of cutaneous and aesthetic surgery. 2012;5(3):163.

9. Fabi SG, Goldman MP. Hand rejuvenation: a review and our experience. Dermatologic Surgery. 2012;38 (7pt2):1112-27.

10. Streker M, Reuther T, Krueger N, Kerscher M. Stabilized hyaluronic acid-based gel of non-animal origin for skin rejuvenation: face, hand, and decolletage. Journal of drugs in dermatology: JDD. 2013;12(9):990-4.

11. Sadick NS. A 52-week study of safety and efficacy of calcium hydroxylapatite for rejuvenation of the aging hand. Journal of drugs in dermatology: JDD. 2011;10(1):47-51.

12. Redaelli A. Cosmetic use of polylactic acid for hand rejuvenation: report on 27 patients. Journal of cosmetic dermatology. 2006;5(3):233-8.

13. Teimourian B, Adham MN. Rejuvenation of the hand: fat injection combined with TCA peel. Aesthetic Surgery Journal. 2000;20(1):70-1.

14. Aust MC, Reimers K, Repenning C, Stahl F, Jahn S, Guggenheim $\mathrm{M}$, et al. Percutaneous collagen induction: minimally invasive skin rejuvenation without risk of hyperpigmentation-fact or fiction? Plastic and reconstructive surgery. 2008;122 (5):1553-63.

15. Fournier PF. Fat transfer to the hand for rejuvenation. Autologous Fat Transfer: Springer; 2010. p. 273-80.

16. Wendt JR. Distal, dorsal superior extremity plasty. Plastic and reconstructive surgery. 2000;106(1):2103 .

17. Brandt FS, Cazzaniga A, Strangman N, Coleman J, Axford-Gatley R. Long-Term Effectiveness and Safety of Small Gel Particle Hyaluronic Acid for Hand Rejuvenation. Dermatologic Surgery. 2012;38 (7pt2):1128-35.

18. Alster TS, Konda S. Plasma skin resurfacing for regeneration of neck, chest, and hands: investigation of a novel device. Dermatologic Surgery. 2007;33 (11):1315-21.

19. Aust M, Knobloch K, Gohritz A, Vogt PM, Fernandes D. Percutaneous collagen induction therapy for hand rejuvenation. Plastic and reconstructive surgery. 2010;126(4):203e-4e.

20. Bank J, Fuller SM, Henry GI, Zachary LS. Fat grafting to the hand in patients with Raynaud phenomenon: a novel therapeutic modality. Plastic and reconstructive surgery. 2014;133(5):1109-18.

21. Bidic SM, Hatef DA, Rohrich RJ. Dorsal hand anatomy relevant to volumetric rejuvenation. Plastic and reconstructive surgery. 2010;126(1):163-8.

22. Zuk PA, Zhu M, Mizuno H, Huang J, Futrell JW, Katz AJ, et al. Multilineage cells from human 
adipose tissue: implications for cell-based therapies. Tissue engineering. 2001;7(2):211-28.

23. Mizuno H, Tobita M, Uysal AC. Concise review: adipose-derived stem cells as a novel tool for future regenerative medicine. Stem cells. 2012;30(5):80410.

24. Lim MH, Ong WK, Sugii S. The current landscape of adipose-derived stem cells in clinical applications. Expert reviews in molecular medicine. 2014; $16:$ e8

25. Coleman SR. Structural fat grafting: more than a permanent filler. Plastic and reconstructive surgery. 2006;118(3S):108S-20S.

26. Fournier PF. Who Should Do Syringe Liposculpturing? : LWW; 1988.

27. Butterwick KJ. Lipoaugmentation for aging hands: A comparison of the longevity and aesthetic results of centrifuged versus noncentrifuged fat.
Dermatologic surgery. 2002;28(11):987-91.

28. Sommer B, Sattler G. Current concepts of fat graft survival: histology of aspirated adipose tissue and review of the literature. Dermatologic Surgery. 2000;26(12):1159-66.

29. Carpaneda CA, Ribeiro MT. Percentage of graft viability versus injected volume in adipose autotransplants. Aesthetic plastic surgery. 1994;18 (1):17-9.

30. Vara AD, Miki RA, Alfonso DT, Cardoso R. Hand fat grafting complicated by abscess: a case of a bilateral hand abscess from bilateral hand fat grafting. Hand. 2013;8(3):348-51.

31. Kokai LE, Marra K, Rubin JP. Adipose stem cells: biology and clinical applications for tissue repair and regeneration. Translational Research. 2014;163 (4):399-408. 